\title{
200 Jahre Wiener Universitätspathologie
}

\author{
Roland Sedivy $\cdot$ Walter Feigl
}

Eingegangen: 13. Mai 2013 / Angenommen: 14. Mai 2013 / Online publiziert: 18. Juni 2013

(C) Springer-Verlag Wien 2013

Dieser Themenschwerpunkt entstand anlässlich der zweihundertsten Wiederkehr der Restitution der Wiener Pathologie im Jahre 1812. Ende des Jahres, am 12.12.12 fand daher $\mathrm{zu}$ diesem Thema ein wissenschaftlicher Abend in der Wiener Gesellschaft der Ärzte statt. Die Pathologie ist ein immerwährendes Thema der Medizin und sie ändert sich in der Zeit, die an einen heraklidischen Fluss erinnert, in dem wir in unserem Boot versuchen, das platonische Meer des Wissens zu erreichen. Solches verlangt von Zeit zu Zeit eine Standortbestimmung und dazu dienen Jubiläumsveranstaltungen. Daher danken wir sowohl der Gesellschaft der Ärzte wie auch der WMW, dass sie das Vorhaben, 200 Jahre Pathologiegeschichte nachzuzeichnen, spontan und tatkräftig unterstützt haben. Den heutigen Protagonisten des Faches Pathologie zeigt es überdies, wer aller uns geholfen hat, wer uns behindert hat, den genommenen Weg einzuschlagen, und macht uns letztlich dankbar. Es hilft die eigene gegenwärtige Position zu erkennen, es lässt uns das Erfahrene integrieren und damit aus Vergangenem zu lernen.

Den Vorsitz der Abendveranstaltung hatten der PastPräsident der Österreichischen Gesellschaft für Pathologie Andreas Chott (Wilhelminenspital Wien) und der Pathologieordinarius Dontscho Kerjaschki, der auch den Abend einleitete. Mit Grußworten von Frau Druml, der Vizerektorin der Wiener Medizinischen Universität, die

Prim. Univ.-Prof. Dr. R. Sedivy, MD, PhD, MLS ( $\square)$

Institut für Pathologie, LK St. Pölten,

Propst Führer Str. 3-4, 3100 St. Pölten, Österreich

E-Mail: roland.sedivy@meduniwien.ac.at

Prim. Univ.-Prof. Dr. R. Sedivy, MD, PhD, MLS

Zentrum für Pathologie, Danube Private University,

$3500 \mathrm{Krems} /$ Donau, Österreich

Univ.-Prof. DDr. Walter Feigl

Institut für Klinische Pathologie, Medizinische Universität Wien das Department für Geschichte der Medizin leitet, wurde auch schon das Thema angesprochen. Sie hob in ihrem Referat die Pathologisch-anatomischen Sammlungen im Narrenturm als Kulturschatz hervor, der nicht nur zur Didaktik seiner Zeit beitrug, sondern heute Forschungstüren in die Vergangenheit eröffnet. Dem kann nur hinzugefügt werden, dass eine solche Forschung auch heute - und gerade heute wieder durchgeführt wird [1, 2, 3]. Es konnte sogar gezeigt werden, dass diese Exponate angesichts der aktuellen molekularbiologischen Diagnostik morphologische Einblicke in die Pathogenese von Tumoren mit deren genetischen Profilen erlauben [1].

Der eigentliche Programmbeginn war die Chronologie der Wiener Pathologie über 200 Jahre in einer Zeitreise in 20 min von einem der Autoren dieses Editorals (Sedivy). Elf Ordinarii sind es bisher. Es begann mit dem großen Rokitansky, setzte sich fort mit seinen Schülern Heschl und Kundrat, dem herausragenden Weichselbaum um die Jahrhundertwende; es folgten kurze Amtszeiten von Albrecht und Kolisko, danach Maresch mit seiner legendären Schule; daraus Chiari, sein Schüler wiederum Holzner; nach dessen Emeritierung folgte nach einer kurze Amtszeit von Radaszkiewicz der momentane Amtsinhaber Kerjaschki. Detailliert findet sich dies in der Einleitungsarbeit dieser Ausgabe. Christian Köberl, Direktor des Naturhistorischen Museums in Wien, referierte danach über die Bedeutung der pathologisch-anatomischen Sammlung im Narrenturm, die seit Kurzem in das große Museum eingegliedert wurde. Ihm folgte Giulio Superti-Furga, der wissenschaftliche Leiter des Forschungszentrums für Molekulare Medizin (CeMM) und dieser hielt fest: „Die Zukunft liegt in der Vernetzung der modernsten Methoden. Die Erforschung von Krankheitsmechanismen werde in Zukunft ,prädelektiv' (vorhersagend), ,personifiziert', ,präventiv‘ und ,partizipatorisch' sein." [4] Die Pathologie hat somit seit der Restitution eine enorme Wandlung vollzogen. Stand einst die Obduktion im Vordergrund, so sind molekulare 
Diagnosen für die heutige Pathologie unerlässlich für die zielgerichtete Therapie lebender Menschen. Der Abend wurde von Kerjaschki beschlossen, der nochmals auf die Bedeutung des Faches hinwies. „Wir sind eines der größten Institute Europas mit 200 Beschäftigten, 36 Arztpositionen und neun Ausbildungsstellen. Wir erstellen die Diagnosen für 50.000 Patienten pro Jahr", schilderte er [4]. Sein Lehrer, J. Heinrich Holzner, der genannte Emeritus der Wiener Pathologie, durfte an diesem Abend noch anwesend sein. Er erhielt spontanen Applaus, als der heutige Vorstand des Instituts, Dontscho Kerjaschki, seine Leistungen würdigte. Holzner ist im Jänner 2013 von uns gegangen - ein kurzer Nachruf ist diesem Heft beigefügt.

Im vorliegenden Heft werden noch zwei ganz verschiedene Vertreter des Faches Pathologie aus sehr verschiedenen Zeiten gewürdigt. Lorenz Biermayer war der Pathologe zur Zeit der Restitution 1812, er war der erste außerordentliche Professor. Auf ihn geht eine Neuordnung des Museums zurück (Arbeit Patzak et al. in dieser Ausgabe), desgleichen ist er der Begründer der seit 1817 durchgehend erhaltenen Sammlung der Wiener Obduktionsbefunde (Arbeit Winter et. al). Alfons Portele, ein Chiari-Schüler, betreute das Pathologisch-anatomische Museum und erweiterte es zu einem der weltweit
Größten seiner Art (Arbeit Patzak und Winter). Gerade die Beschäftigung mit der Geschichte lässt unser eingangs erwähntes Boot immer zügiger, immer geschickter, immer eleganter vorankommen. Aber zwischendurch kreuzen wir auch gegen den Strom - denken zurück und manchmal staunen wir. Damit dürfen wir uns den Wünschen für die Pathologie anschließen - ad multos annos!

\section{Interessenkonflikt}

Es besteht kein Interessenkonflikt.

\section{Literatur}

1. Sedivy R, Kalipciyan M, Patzak B, Mader RM. KRAS mutations in historical tumour specimens of the Viennese Museum of pathological anatomy. Histopathology. 2011;58(5):792-6.

2. Sedivy R. Pathologie in Fallstudien. Historische Präparate neu betrachtet. New York: Springer; 2007.

3. Sedivy R, Patzak B. Pancreatic diseases past and present: a historical examination of exhibition specimens from the Collectio Rokitansky in Vienna. Virchows Arch. 2002;441(1):12-8.

4. http://derstandard.at/1353209115351/200-Jahre-Institutfuer-Pathologie-in-Wien. zitiert 12.5.2013 